\title{
Mikrolitermethode zur flammenphotometrischen Bestimmung von Natrium, Kalium und Calcium, mit handelsüblichen Geräten
}

\author{
Von D. Stamm und R. Herrmann \\ Aus der Klinisch-chemischen Abteilung (Leiter: Dr. Dr. D. Stamm) an der Chirurgiscben Klinik und aus der Abteilung für \\ Medizinische Pbysik (Leiter: Prof. Dr. R. Herrmann) an der Hautlelinik der Justus-Liebig-Universität Gießen
}

(Eingegangen am 30. Juli 1965)

\begin{abstract}
Es wird über ein flammenphotometrisches Mikroliterverfahren zur Bestimmung von Natrium, Kalium und Calcium im Serum berichtet, das nur 30 Mikroliter Serum erfordert. Die Reproduzierbarkeit des Verfahrens ist ebensogut wie die herkömmlichen Milliliterverfahren mit geeichtem Gerät, aber besser als die Millilitermethoden mit eichfähigem Gerät, wie es üblicherweise bei Routineuntersuchungen verwendet wird. Das Verfahren wird geprüft und die Vorzüge diskutiert.
\end{abstract}

\begin{abstract}
A flame photometric method is described for the determination of sodium, potassium and calcium in serum. Only 30 microlitres of serum are required. The reproducibility of the method is as good as that of the customary millilitre method with calibrated apparatus, but better than that of the millilitre method with the adjustable apparatus as normally used for routine studies. The method is tested and its advantages discussed.
\end{abstract}

Bei Säuglingen, besonders bei Frühgeborenen, bereitet es häufig ernste Schwierigkeiten, die für die herkömmlichen Serumelektrolyt-Bestimmungsmethoden notwendigen Blutmengen zu gewinnen. Wenn man einem Säugling einen Milliliter Blut entnimmt, so entspricht das einer Blutentnahme von 18 Millilitern beim Erwachsenen. Bei optimaler Ausnutzung würde man für eine Elektrolytbestimmung im herkömmlichen Maßstabe etwa $3 \mathrm{~m} l$ Blut benötigen, das entspräche rund $60 \mathrm{ml}$ beim Erwachsenen. Man hat darum im Laufe der letzten Jahre immer wieder Mikroverfahren entwickelt (1-4), die aber besondere Apparaturen erfordern, für deren Bedienung eine spẹzielle Úbung nötig ist. Diese Mikroverfahren haben sich deshalb nicht als Routineuntersuchungen eingeführt.

Seit neuester Zeit steht ein leicht $\mathrm{zu}$ handhabendes Mikrolitersystem ${ }^{1}$ ) zur Verfügung (5), dessen Kernstück Kolbenhubpipetten, sogenannte Marburg-Pipetten, sind, die auch von ungeübten technischen Assistentinnen gern benutzt werden und gut reproduzierbare Ergebnisse liefern. Es erscheint sinnvoll, mit diesem System unter Verwendung eines handelsüblichen Flammenphotometers ${ }^{2}$ ) ein Mikroliterverfahren zu entwickeln, das ohne wesentliche Umbauten an dem Photometer in jedem Routinelaboratorium ausgeführt werden kann.

Nach den hier durchgeführten Untersuchungen können in 30 Mikroliter Serum mit hinreichender Genauigkeit Natrium, Kalium und Calcium flammenphotometrisch bestimmt werden. Die Reproduzierbarkeit der Mikrolitermethode ist im Routinebetrieb mindestens ebensogut, wenn nicht besser als die der Millilitermethode. Die Bestimmung von Chlorid ist durch potentiometrische Titration in 10 Mikroliter Serum mit guter Reproduzierbarkeit möglich. Darüber wird an anderer Stelle berichtet. Für die Bestimmung von Standardbicarbonat

1) Mikrolitersystem „Eppendorf““; Hersteller: Eppendorf-Gerätebau, Netheler u. Hinz GmbH, Hamburg.

$\left.{ }^{2}\right)$ Hersteller: Eppendorf-Gerätebau, Netheler u. Hinz GmbH, Hamburg. und des $\mathrm{pH}$ im arteriellen Blut hat sich das Mikroverfahren von Astrup, das mit Kapillarblut ausgeführt wird, bestens bewährt. Siggard-ANDERSEN (6) hat darüber kürzlich zusammenfassend berichtet.

Wir besitzen danach für akute Notfälle leistungsfähige Mikrolitermethoden zur Bestimmung aller für Diagnose und Therapie wichtigen Elektrolytkonzentrationen im Blut. - Úber unser methodisches Vorgehen bei der Bestimmung der Elemente Natrium, Kalium und Calcium und die Prüfung dieses Verfahrens soll im folgenden berichtet werden.

\section{Methoden \\ Probevorbereitung}

Das Venenblut soll unmittelbar nach dem Einstich aus der ungestauten Vene entnommen werden. Wenn Blut auch für andere Bestimmungen entnommen wird, dann sollte man nur die erste Probe für die Elektrolytbestimmung (7) verwenden. - Blutkuchen und Serum müssen spätestens eine Stunde nach der Entnahme durch Zentrifugieren getrennt werden, da sonst ein beträchtlicher Teil des Kaliums aus den Erythrozyten in das Serum diffundiert und so das Ergebnis verfälscht wird. Hämolytische Seren sind für Elektrolytbestimmungen, besonders für Kaliumanalysen, ungeeignet.

Für jede Probe werden zwei „Reaktionsgefäße (1) mit jeweils $1500 \mu l$ Verdünnungslösung von Zimmertemperatur nach HERRMANN (Merck ,'Titrisol“ Nr. 9975; das Konzentrat muß nach Vorschrift verdünnt werden) beschickt und jeweils $15 \mu /$ Serum hinzugegeben. Die Gefäße werden mit den Kappen fest verschlossen und auf dem Rüttler $\left.{ }^{1}\right) 3$ Minuten gemischt. Wenn man die Vorratsbeutel der „Reaktionsgefäße“ und „Pipettenspitzen“") immcr gut verschlossen hält, erübrigt sich eine vorherige Reinigung der Gcfäße und Spitzen.

\section{Apparat}

Die Messung erfolgt mit dem Flammenphotometer „Eppendorf“, wozu ein von dem Gerätehersteller bezogencr, besonders leistungsfähiger Multiplier sowie ein ausgesuchtes neues Filter für Calcium eingesetzt und eine hohe Empfindlichkeit eingestellt wird. Uns bewährten sich die in der Tabelle 1 angegebenen Einstellungen. Im Prinzip sind auch andere entsprechend ausgerüstete Flammenphotometer geeignet.

Wir benutzen die von dem Hersteller eingebauten Interferenzfilter und zwar für Natrium $589 \mathrm{~m} \mu$, Kalium $767 \mathrm{~m} \mu$ und Calcium 
Tab. 1

Einstellung des Flammenphotometers bei der Mikrolitermethode

\begin{tabular}{|c|c|c|c|c|c|c|}
\hline \multirow{3}{*}{ Element } & \multicolumn{2}{|c|}{ Preßluft $\left.{ }^{1}\right)$} & \multicolumn{2}{|c|}{ Brenngas } & \multicolumn{2}{|c|}{ Multiplier } \\
\hline & $\mathrm{kg} / \mathrm{cm}^{2}$ & l/Min. & Art u. Druck & & & Spannung \\
\hline & & & in $\mathrm{mmH}_{2} \mathrm{O}$ & l/Min. & Stufe & V \\
\hline Natrium & 0,50 & 4 & Propan 210 & 0,30 & $3-4$ & $500-580$ \\
\hline Kalium & 0,50 & 4 & Propan 210 & 0,30 & 9 & 1000 \\
\hline Calcium & 0,50 & 4 & $\begin{array}{l}\text { Acetylen } \\
460-500\end{array}$ & 0,35 & 9 & 1000 \\
\hline
\end{tabular}

1) Der Schutzgasfluß um die Flamme ist hierbei nicht berücksichtigt; er beträgt zusätzlic̣h etwa 5 l/Min.

$622 \mathrm{~m} \mu$. - Zur Verkürzung der Einstellzeit wird der Ansaugschlauch an der Kapillare des Indirektzerstäubers auf $8 \mathrm{~cm}$ verkürzt; auf das sonst vorgeschaltete Ansaugsieb wird verzichtet. Die Einstellzeit des Gerätes beträgt dann 16-18 Sekunden. Pro Minute werden bei dieser Einstellung $1200 \mu l$ angesaugt. Unter Einstellzeit versteht man diejenige Zeit, die vom Eintauchen des Ansaugschlauches in die Probe bis zum Erreichen von $99 \%$ des Endausschlages vergeht.

\section{Eicbung}

Zur Eichung wird die von HerrmanN angegebene Haupteicblösung (Merck „Titrisol“ Nr. 9976) verwendet. Sie enthält 143,5 mval/l Natrium, 3,93 mval/l Kalium und 5,05 mval/l Calcium, also dem Serum entsprechende Elektrolytkonzentrationen. Zur Eichung $\mathrm{mu} ß$ sie in demselben Verhältnis verdünnt werden wie die $z u$ messenden Serumproben. Die größte Meßgenauigkeit wird erzielt, wenn man die Eichlösung mit denselben Pipetten ansetzt, die später auch für die Probenvorbereitung verwendet werden.

Als Eichlösung für alle drei Elemente dient folgende Verdünnung der Haupteichlösung:

In einen $100 \mathrm{~m} / \mathrm{Me}$ ßkolben füllt man bis zur Marke Verdünnungslösung und gibt mit einer $1 \mathrm{~m} /$ Vollpipette $1 \mathrm{~m} /$ Haupteichlösung hinzu. Als Blindlösungen dienen für die Bestimmung von Natrium Verdünnungslösung, für die Bestimmung von Kalium $100 \mathrm{~m} l$ Verdünnungslösung plus $1 \mathrm{~m} l$ Kalium-Blindlösung (Merck „Titrisol“ Nr. 9977) und für die Bestimmung von Calcium $100 \mathrm{ml}$ Verdünnungslösung plus $1 \mathrm{~m} /$ Calcium Blindlösung (Merck „Titrisol“ Nr. 9978). Die beiden letzten Verdünnungen werden in derselben Weise wie die Haupteichlösungsverdünnung hergestellt. Vor jeder Meßreihe werden mit den oben angegebenen Verdünnungen der Haupteichlösung die Eichpunkte und die Nullpunkte für Natrium mit Verdünnungslösung, für Kalium und Calcium mit den Verdünnungen der entsprechenden Blindlösungen eingestellt. Es empfiehlt sich, diesen Eichvorgang nach jeweils 5 Messungen zu wiederholen und nötigenfalls nachzustellen.

\section{Messung}

Die Einstellung der Gaszufuhren zur Flamme des Flammenphotometers erfolgt entsprechend Tabelle 1. Die Einstellung der Nullund Eichpunkte erfolgt beim Verstäuben der jeweiligen Blind- und Eichlösungen. Die Proben bleiben während der Messung fest im Arbeitsblock des Mikrolitersystems ${ }^{1}$ ) sitzen. Die Ablesung der Werte kann spätestens nach 20 Sekunden erfolgen. Gemessen wird also nach dem Ausschlagverfahren, geeicht also mit der Nachstellmethode.

Schleppende Einstellung oder starke Schwankungen am Meßpunkt sind häufig auf Verunreinigungen der Düse oder der Zerstäuberkammer zurückzuführen; beide bedürfen bei dem Mikroliterverfahren einer besonders sorgfältigen Pflege. Da man das Sieb in der Ansaugkapillare weggenommen hat, können feine Fibrinfädchen, die auch nach dem Zentrifugieren der Blutprobe im Serum suspendiert bleiben, die Düse verstopfen. An der Aufprallglasplatte des Vorkammerzerstäubers können sich Serum-Lipide niederschlagen. Beide Verunreinigungen führen zu einer Verlängerung der Einstellzeit. Bei der Mikrolitermethode muß man spätestens nach 20 Se- kunden ablesen können, hingegen steht bei der Millilitermethode genügend Serumverdünnung zur Verfügung, um auf die Einstellung $\mathrm{zu}$ warten.

\section{Prüfung der Methode \\ Reproduzierbarkeit der Geräteanzeige}

Die Reproduzierbarkeit der Messungen durch das Gerät (ohne Einbeziehung des Pipettierfehlers) wird geprüft, indem man einen $100 \mathrm{ml}$ Meßkolben bis zur Marke mit Verdünnungslösung füllt und dann mit einer $1000 \mu l$ Marburg-Pipette ${ }^{1}$ im Ansatz a) Haupteichlösung und im Ansatz b) ein Mischserum hinzugibt. Aus jedem dieser Ansätze werden jeweils 25 Messungen für Natrium, Kalium und Calcium vorgenommen. Daraus werden die Mittelwerte und der mittlere quadratische Fehler berechnet. Die Ergebnisse sind in Tabelle 2 zusammengestellt.

Tab. 2

Reproduzierbarkeit der Geräteanzeige

\begin{tabular}{lcc}
\hline & $\begin{array}{c}\text { a) Haupteichlösung } \\
\text { mval } / l\end{array}$ & $\begin{array}{c}\text { b) Mischserum } \\
\text { mval } / l\end{array}$ \\
\hline Natrium & $143,1 \pm 0,1$ & $150,5 \pm 0,5$ \\
Kalium & $3,90 \pm 0,02$ & $3,19 \pm 0,03$ \\
Calcium & $5,00 \pm 0,01$ & $4,88 \pm 0,05$ \\
. & & \\
\hline
\end{tabular}

\section{Reproduzierbarkeit der Mikrolitermetbode}

Um die Reproduzierbarkeit der Geräteanzeige einschließlich der vorgeschalteten Verdünnungsschritte zu überprüfen, werden entsprechend der Probenvorbereitung in getrennten Reaktionsgefäßen zweimal $25 \mathrm{An}$ sätze mit jeweils $1500 \mu l$ Verdünnungslösung und $15 \mu l$ Haupteichlösung und in einer zweiten Reihe mit jeweils $15 \mu l$ Mischserum mit $1500 \mu l$ Verdünnungslösung vorbereitet. - In allen drei Reihen werden die Konzentrationen von Natrium, Kalium und Calcium entsprechend der obigen Vorschrift gemessen. Nach jeder fünften Probe wird zwischengeeicht und nachgestellt. Zur Erzielung einer konstanten Flammentemperatur empfiehlt es sich, das Gerät eine Viertelstunde mit Haupteichlösungsverdünnung einbrennen $\mathrm{zu}$ lassen.

Aus den jeweils 25 Ablesungen für jedes Element werden Mittelwert und mittlerer quadratischer Fehler berechnet, die der Tabelle $3 \mathrm{zu}$ entnehmen sind.

Bei Ansatz a) werden die $15 \mu l$ Mischserum mit einer $5 \mu$-Pipette und einer $10 \mu l$-Pipette nacheinander hin- 
Tab. 3

Reproduzierbarkeit der Mikrolitermethode

\begin{tabular}{lccc}
\hline & $\begin{array}{c}\text { Haupteichlösung } \\
\text { mval } / l\end{array}$ & $\begin{array}{c}\text { Mischserum } \\
\text { Ansatz a) } \\
\text { mval } / l\end{array}$ & $\begin{array}{c}\text { Ansatz b) } \\
\text { mval } / l\end{array}$ \\
\hline Natrium & $143,5 \pm 0,3$ & $147,8 \pm 0,56$ & $146,4 \pm 0,3$ \\
Kalium & $3,92 \pm 0,02$ & $4,13 \pm 0,12$ & $4,23 \pm 0,03$ \\
Calcium & $5,03 \pm 0,01$ & $4,67 \pm 0,03$ & $4,84 \pm 0,02$ \\
\hline
\end{tabular}

zugegeben. Bei dem Ansatz b) erfolgt die Probenzugabe auf einmal mit einer $15 \mu \mathrm{l}$-Pipette, einer Sonderanfertigung für die Elektrolytbestimmung ${ }^{2}$ ).

\section{Reproduzierbarkeit der Millilitermethode}

Die Geräteeinstellung erfolgt nach den Angaben des Herstellers, die wir in der Tabelle 4 wiederholen. Die Filter sind dieselben wie bei der Mikrolitermethode.

Die Eichlösungen werden nach einer Vorschrift des Geräteherstellers aus Konzentraten hergestellt. In einen $200 \mathrm{ml}$ Meßkolben gibt man $10 \mathrm{ml}$ Standardlösung2) (Natrium 143,5 mval/l, Kalium 3,84 mval/l, Calcium $5,00 \mathrm{mval} / l)$, fügt $10 \mathrm{~m} /$ Netzmittellösung ${ }^{2}$ ) hinzu und füllt mit bidestilliertem Wasser bis zur Marke auf: Lösung I. Diese Lösung I wird zur Eichung für Kalium und Calcium verwendet. Die Natrium-Eichlösung wird aus Lösung I hergestellt; man gibt in einen $200 \mathrm{ml}$ Meßkolben $20 \mathrm{~m} l$ Lösung I, fügt $10,0 \mathrm{~m} /$ Netzmittellösung2) hinzu und füllt bis zur Marke mit bidestilliertem Wasser auf. Die Einstellung des Nullpunktes für Natrium und Kalium erfolgt beim Ansaugen von bidestilliertem Wasser; bei Calcium verwendet man eine Kompensationslösung, die aus $10 \mathrm{~m} l$ Konzentrat $\left.{ }^{2}\right), 10 \mathrm{~m} l$ Netzmittel ${ }^{2}$ ) in einem $200 \mathrm{ml}$ Meßkolben durch Auffüllen mit bidestilliertem Wasser hergestellt wird.

\section{Prüfung der Linearität der Eichlearven}

$\mathrm{Da}$ es durchaus möglich ist, $\mathrm{da} B$ bei der stärkeren Verdünnung der Meßlösungen für die Bestimmung von Kalium von Calcium und der schwächeren Verdünnung für Natrium die Eichkurven in dem interessierenden Bereich z. B. durch Ionisationseinflüsse oder infolge Selbstabsorption nicht linear sind, wird dies überprüft. Es ist auch eine gegenseitige Beeinflussung von Natrium und Kalium und von Natrium und Calcium bekannt. Darum erfolgt die Aufstellung der Eichkurven mit
Tab. 5

Reproduzierbarkeit der Millilitermetbode; Werte in mval/l.

\begin{tabular}{lccc}
\hline Element & $\begin{array}{c}\text { Haupt- } \\
\text { cichlösung } \\
\text { geeicht. Gerät }\end{array}$ & $\begin{array}{c}\text { Mischserum } \\
\text { geeicht. Gerät }\end{array}$ & $\begin{array}{c}\text { Mischserum } \\
\text { eichfähiges Gerät } \\
\text { Routinebedingungen }\end{array}$ \\
\hline Natrium & $143,8 \pm 0,5$ & $154,2 \pm 0,5$ & $141,1 \pm 1,0$ \\
Kalium & $3,65 \pm 0,01$ & $4,40 \pm 0,02$ & $4,26 \pm 0,03$ \\
Calcium & $5,14 \pm 0,01$ & $4,80 \pm 0,01$ & $5,13 \pm 0,06$ \\
\hline
\end{tabular}

Modellseren (7a). Diese Modellseren sind wäßrige Lösungen, die Natrium, Kalium und Calcium enthalten. Bei der Herstellung der einzelnen Eichlösungen wird die Konzentration des zu messenden Bestandteils auf die aus den Eichkurven $z u$ ersehenden Werte eingestellt; die Konzentration der übrigen Bestandteile bleibt konstant auf den Werten der Haupteichlösung (Natrium $143,5 \mathrm{mval} / l$, Kalium 3,84 mval/l, Calcium 5,00 mval/ll). In den nachfolgenden Abbildungen 1,2 und 3 sind auf den Abszissen die vorgegebenen Konzentrationen und auf den Ordinaten die flammenphotometrisch ermittelten Werte eingetragen.

Die Eichkurven sind linear und haben bei Wahl gleicher Einheiten auf Abszisse und Ordinate die Steigung 1,00. Es besteht also keine Abweichung, die die Anbringung von Korrekturfaktoren zu den auf der Skala abgelesenen Werten erfordert.

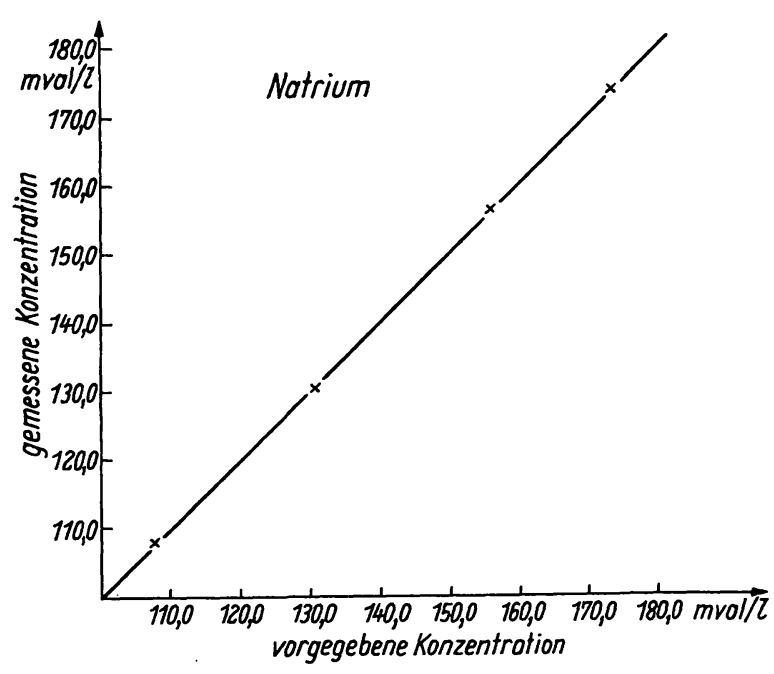

Abb. 1

Eichkurve für Natrium

Tab. 4

Einstellung des Flammenphotometers bei der Millilitermethode

\begin{tabular}{|c|c|c|c|c|c|c|}
\hline \multirow[b]{2}{*}{ Element } & \multicolumn{2}{|c|}{ Preßluft } & \multicolumn{2}{|c|}{ Brenngas } & \multicolumn{2}{|c|}{ Multiplier } \\
\hline & $\mathrm{kg} / \mathrm{cm}^{2}$ & l/Min. & $\begin{array}{l}\text { Art u. Druck } \\
\text { in } \mathrm{mm} \mathrm{H}_{2} \mathrm{O}\end{array}$ & //Min. & Stufe & $\begin{array}{c}\text { Spannung } \\
\mathrm{V}\end{array}$ \\
\hline Natrium & 0,50 & 4 & Propan 210 & 0,30 & 4 & 580 \\
\hline Kalium & 0,50 & 4 & Propan 210 & 0,30 & 4 & 760 \\
\hline Calcium & 0,50 & 4 & Acetylen 400 & 0,28 & 7 & 840 \\
\hline
\end{tabular}




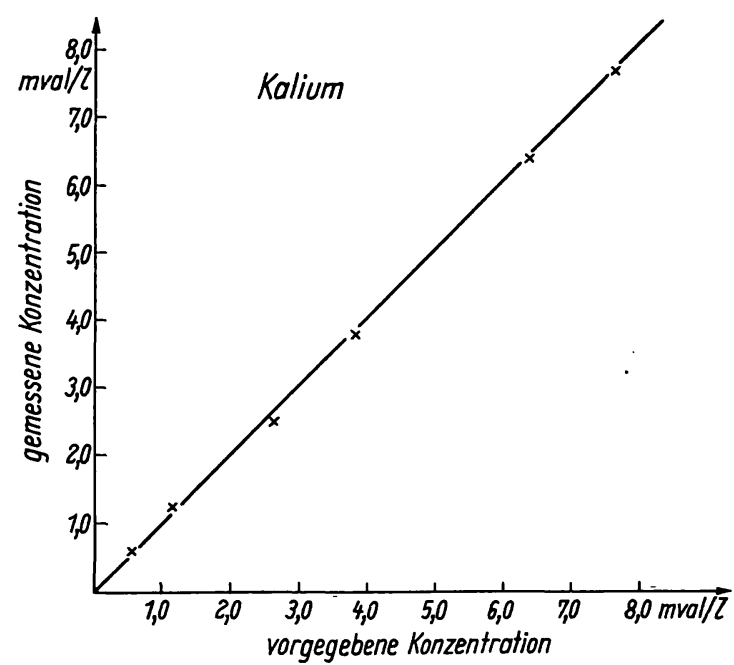

Abb. 2

Eichkurve für Kalium

Parallelbestimmungen mit der Mikroliter- und MilliliterMethode bei Patienten-Seren

Aus dem Serum von 31 Patienten werden Parallelbestimmungen mit der Mikroliter-Methode und der Milliliter-Methode ausgeführt, wobei jeweils von der gleichen Serumprobe ausgegangen wird. Die Mittelwerte mit beiden Methoden und die Erwartungswerte der Streuungen beider Methoden gegeneinander $E_{\text {(Str) }}$ sind in Tabelle 6 eingetragen. Der $E_{(\mathrm{Str})}$ wird mit Hilfe der Beziehung

$$
E_{(\mathrm{Str})}=\sqrt{\frac{\Sigma(\Delta)^{2}}{2 m}}
$$

berechnet, wobei $m$ die Anzahl der Doppelbestimmungen und $\Delta$ die Abweichungen der einzelnen Wertepaare untereinander bedeuten $(8,9)$.

Tab. 6

Parallelbestimmungen mit Mikroliter- und Millilitermethode; (Werte in mval/l).

\begin{tabular}{lccc}
\hline Element & \multicolumn{2}{c}{ Mittelwerte } & Erwartungswert \\
Milliliter- & $\begin{array}{c}\text { Mikroliter- } \\
\text { methode }\end{array}$ & $\begin{array}{c}\text { der } \\
\text { Streuung }\end{array}$ \\
\hline Natrium & 149,3 & 149,3 & $2,8(=2 \%)$ \\
Kalium & $4,2_{3}$ & $4,2_{1}$ & $0,16(=4 \%)$ \\
Calcium & $4,4_{3}$ & $4,4_{5}$ & $0,16(=4 \%)$ \\
\hline
\end{tabular}

\section{Diskussion}

Die vorliegenden Untersuchungen zeigen, daß die beschriebene Mikrolitermethode für die flammenphotometrische Bestimmung von Natrium, Kalium und Calcium im Routinelaboratorium sehr gut geeignet ist. Gegenüber den bisher üblichen Milliliterverfahren hat sie folgende Vorteile:

1. Der Verbrauch an Untersuchungsgut ist wesentlich geringer. Das Mikroliterverfahren benötigt nur $30 \mu l$ Serum gegenüber $1000 \mu l$ bei dem Milliliterverfahren.

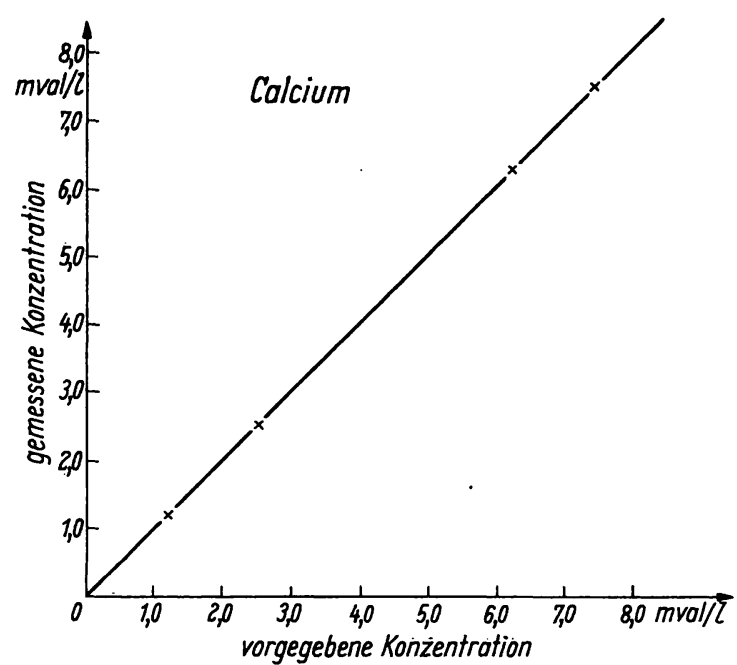

Abb. 3

Eichkurve für Calcium

2. Verunreinigungen der Geräte beim Spülen sind nicht möglich, da man für jeden Ansatz stets neue Reaktionsgefäße und Pipettenspitzen nehmen kann, die so billig sind, daß man sie nach Gebrauch wegwirft.

3. Das Verfahren ist billiger als die herkömmliche Millilitermethode, weil man einerseits den Arbeitsaufwand für das Spülen der Geräte und den dabei auftretenden beträchtlichen Glasbruch einspart, andererseits werden die Meßzeiten wesentlich kürzer. Dadurch wird auch Arbeitszeit der technischen Assistentinnen eingespart. Die Zeiten für das genaue Pipettieren mit den Kolbenhubpipetten des Mikrolitersystems sind wesentlich kürzer als bei dem Pipettieren mit dem Glasgerät, da die Zeiten für das Aufsaugen, das Einstellen des Meniskus auf die Marke, die in der Eichvorschrift vorgeschriebene Auslaufzeit und Wartezeit, die zusammen etwa eine Minute betragen, wesentlich länger sind als eine Kolbenhubpipettierung. Neben der Einsparung an Arbeitszeit spart man auch Gas und Strom und Betriebsstunden des Gerätes bei gleichem Probendurchlauf.

4. Die Infektionsmöglichkeit des Personals beim Pipettieren und Spülen ist wesentlich geringer als bei dem Milliliterverfahren.

5. Die Reproduzierbarkeit des Mikroliterverfahrens ist, wie ein Vergleich der Tabellen 3 und 5 ergibt, genau so gut wie die des Milliliterverfahrens mit geeichtem Gerät. Bei Routineuntersuchungen verwendet man aber im allgemeinen wegen der hohen Kosten nur ein eichfähiges Gerät. Wie unsere Vergleichsuntersuchungen in den Tabellen 3 und 5 zeigen, ist die Reproduzierbarkeit der Mikrolitermethode in der Routine besser als die der Millilitermethode mit eichfähigem Gerät.

Der hier durchgeführte Vergleich sagt jedoch noch nichts über die Richtigkeit (accuracy), denn es wird hier ein flammenphotometrisches Verfahren mit einem anderen flammenphotometrischen verglichen. Beide Verfahren stellen, wie alle spektrochemischen Methoden, nur 
Relativverfahren dar und bedürfen einer Eichung. Da man für die Eichung aber Lösungen verwendet, die nicht in allen Eigenschaften den natürlichen Proben entsprechen, sind systematische Fehler denkbar, die sich für beide Verfahren in gleicher Weise auswirken können.
Die Ermittlung solcher systematischer Fehler für die vorliegenden Verhältnisse wird zur Zeit bearbeitet; darüber soll in einer späteren Mitteilung berichtet werden.

Frau I. Hofmerster danken wir für die sorgfältige Durchführung der flammenphotometrischen Messungen.

\title{
Literatur
}

1. Herrmann, R., Zschr. exper. Med. 118, 187 (1952). - 2. HerrMANN, R. und R. BAUMANN, Zschr. exper. Med. 119, 487 (1952). 3. BaumanN, R. und R. HerrmanN, Zschr. exper. Med. 120, 172 (1953). - 4. HerrmanN, R., Zschr. exper. Med. 120, 84 (1953). 5. Bürtrner, H., Dtsch. med. Wschr. 88,910 (1964). - 6. SigGAARD-ANDERSEN, O., The acid-base status of the blood, Munks-
gaArd, Copenhagen (1964). - 7. Herrmann, R. und C. Th. J. Alkemade, Flammenphotometrie, 2. Aufl. S. 305. Springer-Verlag, Berlin-Göttingen-Heidelberg (1960). - 7a. daselbst S. 212. - 8. Gebelein, H. und J. Heite, Statistische Utteilsbildung, Springer-Verlag, Berlin (1951). - 9. Rausch, L. und H. Graul, Ärztl. Wschr. 4, 591 (1949).
Dr. med., Dr. rer. nat. D. Stamm Klinisch-chemische Abteilung an der Chirurgischen Universitätsklinik 63 Gießen, Klinikstraße 37

\section{KURZMIT'TEILUNGEN}

\author{
Beitrag zur Arbeit S. ZimmermanN

\section{Polarographische Untersuchungen der elektrophoretisch aufgetrennten Albuminfraktion}

\author{
Von J. Homolka \\ Aus der Abteilung für klinische Biochemie der medizinischen Fakultät der Karlsuniversität, Prag/CSSR \\ (Vorstand: Professor MUDr. J. Homolka, Dr. Sc.)
}

(Eingegangen am 20. September 1965)

In dieser Zeitschrift (1) berichtete S. ZimmermanN über die polarographische Untersuchung der elektrophoretisch aufgetrennten Albuminfraktion, wobei er eine ursprünglich von НомоLKA angegebene polarographische Methode modifizierte und im allgemeinen schlechte Ergebnisse erhielt.

Im folgenden wird diese Frage diskutiert und gezeigt, weshalb die ZimmermanNsche Modifikation schlechte Resultate gab. In seiner Arbeit diskutiert ZimmermanN die Menge des zur polarographischen Analyse eingesetzten Albumins und den Einfluß der Mucoproteine. Als $\mathrm{Ma}$ der Freisetzung benutzt er die Erhöhung der polarographischen Welle durch Alkalidenaturation, was in meiner Terminologie jedoch nur eine sogenannte relative Freisetzung bedeutet. Schon seit Entdeckung der BRDIčKA-Reaktion ist bekannt, daß die Höhe der Proteinwelle von der Konzentration an Protein nicht linear abhängt, sondern der Langmuir-Isothermen gehorcht. Diese Frage ist mir natürlich bekannt und bereits in einer im Jahre 1953 erschienenen Publikation (2) habe ich diese Konzentrationsabhängigkeit verfolgt. Unter unseren Bedingungen, d. h. bei einer großen Verdünnung ist sie praktisch linear und besitzt keinen Einfluß auf die maximale oder relative Freimachung. In einer neueren Arbeit haben wir weiterhin den Einfluß der Mucoproteine in den Filtraten der Fraktionen polarographisch verfolgt. Diese könnten sich nur bei den $\alpha$-Globulinen in etwa $10 \%$ der Wellenhöhe des $\alpha$-Globulins bemerkbar machen. Unter unseren Meßbedingungen kann der Mucoprotein-Gehalt bzw. seine polarographische Aktivität in der Albuminfraktion vernachlässigt werden (3). Der Mucoprotein-Gehalt kann niemals die gleiche Konzentration wie das Albumin oder - wie bei ZrmmermanN - ein Viertel des Albumins erreichen.

$\mathrm{Da}$ wir die bei der Analyse (Elektrophorese, Austrocknen und Elution) einsetzende Proteindenaturierung nicht vollständig standardisieren können, benutzen wir für die klinische Auswertung nicht die relative Freisetzung, sondern die maximale Freisetzung. Falls es trotz der unbedingt nötigen Aufmerksamkeit bei der Bestimmung vor der Alkalizugabe zu verschiedenen Stufen der Albumindenaturierung kommt, werden die relativen Albuminprozente höher und die relative Freimachung kleiner gefunden. Wir benutzen daher jedesmal das Produkt dieser beiden Werte, d. h. die maximale Freisetzung des Albumins (3). Gerade dieser Wert ist der empfindlichste Indikator für den jeweiligen klinischen Zustand.

Weiterhin benutzen wir für polarographische Untersuchungen als Standard niemals das Blut gesunder Blutspender, da gerade bei ihnen - wie in unserer 